research article

\title{
Postoperative radiochemotherapy for gastric adenocarcinoma: long term results
}

\author{
Irena Oblak, Franc Anderluh, Vaneja Velenik \\ Department of Radiotherapy, Institute of Oncology Ljubljana, Ljubljana, Slovenia
}

Background. To analyze the efficacy of postoperative radiochemotherapy with 5-florouracil (5-FU) and leucovorin (LV) applied in the patients with gastric carcinoma treated in a single institution.

Patients and methods. Between 2001 and 2004, 123 patients with resected gastric adenocarcinoma were treated with postoperative concomitant radiochemotherapy with 5-FU and $L V$. The adjuvant treatment consisted of five cycles of chemotherapy with 5-FU $\left(425 \mathrm{mg} / \mathrm{m}^{2} \mathrm{IV}\right)$ and $\mathrm{LV}\left(20 \mathrm{mg} / \mathrm{m}^{2} \mathrm{IV}\right)$ and concomitant radiotherapy with the total dose of $45 \mathrm{~Gy}$.

Results. The treatment was completed according to the protocol in $82 \%$ of patients. The frequency and severity of early toxic effects induced by radiochemotherapy were manageable. Median follow-up time of 56 survivors was 64.5 months (range: 51.7-96.4 months). The 5-year locoregional control (LRC), diseasefree survival (DFS), disease-specific survival (DSS) and overall survival (OS) were 81\%, 48.3\%, 50.4\%, and $48.4 \%$, respectively. The multivariate analysis showed that the tumor involvement of cardia and low intensity of chemotherapy were independent adverse prognostic factors for DSS and OS. More advanced $p$ T-stage and tumors with diffuse growth type according to Lauren were identified as negative independent prognostic factor for OS. They were also on the threshold of statistical significance for DSS.

Conclusions. Postoperative radiochemotherapy for gastric carcinoma has acceptable toxicity, and is effective particularly in regard to LRC. High incidence of distant metastases calls for more effective systemic regimens.

Key words: gastric cancer; adjuvant therapy; radiochemotherapy; survival; toxicity

\section{Introduction}

Received 21 August 2009

Accepted 28 August 2009

Correspondence to: Assist. Prof. Irena Oblak, MD, PhD, Department of Radiotherapy, Institute of Oncology Ljubljana, Zaloška c. 2, 1000 Ljubljana, Slovenia. Phone: +386 15879 297; Fax: +386 15879 304; E-mail: ioblak@onko-i.si
In Slovenia as well as worldwide, gastric carcinoma is considered as a disease with poor prognosis. Radical surgery which can be performed in only $50-70 \%$ of patients ${ }^{1,2}$ is a common treatment for localized disease. Long-term outcomes with surgery alone are still relatively poor because disease recurrence was observed in $75 \%$ of patients, and in $40-65 \%$ of them, the disease recurred 
Table 1. Pathohistological characteristics of tumors $(n=123)$

\begin{tabular}{|c|c|c|c|}
\hline Characteristics & & No. & $\%$ \\
\hline \multirow[t]{4}{*}{ pT - stage } & 1 & 9 & 7.3 \\
\hline & 2 & 39 & 31.7 \\
\hline & 3 & 66 & 53.7 \\
\hline & 4 & 9 & 7.3 \\
\hline \multirow[t]{4}{*}{$\mathrm{pN}$ - stage } & 0 & 4 & 3.3 \\
\hline & 1 & 53 & 43.1 \\
\hline & 2 & 42 & 34.1 \\
\hline & 3 & 24 & 19.5 \\
\hline \multirow[t]{5}{*}{ Overall stage } & $\mathrm{Ib}$ & 6 & 4.9 \\
\hline & II & 26 & 21.1 \\
\hline & IIIa & 37 & 30.1 \\
\hline & $\mathrm{IIIb}$ & 24 & 19.5 \\
\hline & IV & 30 & 24.4 \\
\hline \multirow[t]{4}{*}{ Pathohistological tumor grade } & 1 & 5 & 4.1 \\
\hline & 2 & 25 & 20.3 \\
\hline & 3 & 87 & 70.7 \\
\hline & unknown & 6 & 4.9 \\
\hline \multirow[t]{5}{*}{ Bormann type } & 1 & 4 & 3.3 \\
\hline & 2 & 14 & 11.4 \\
\hline & 3 & 43 & 35 \\
\hline & 4 & 24 & 19.5 \\
\hline & unknown & 38 & 30.9 \\
\hline \multirow[t]{3}{*}{ Growth type according to Lauren } & diffuse & 61 & 49.6 \\
\hline & intestinal & 55 & 44.7 \\
\hline & unknown & 7 & 5.7 \\
\hline \multirow[t]{3}{*}{ Perineurial invasion } & yes & 59 & 48 \\
\hline & no & 45 & 36.6 \\
\hline & unknown & 19 & 15.4 \\
\hline \multirow[t]{3}{*}{ Lymphovascular invasion } & yes & 66 & 53.7 \\
\hline & no & 23 & 18.7 \\
\hline & unknown & 34 & 27.6 \\
\hline \multirow[t]{3}{*}{ Angioinvasion } & yes & 23 & 18.7 \\
\hline & no & 45 & 36.6 \\
\hline & unknown & 55 & 44.7 \\
\hline
\end{tabular}

Data on late side effects are scarce. It is suspected that the used radiation fields can cause the damage to the left kidney in some patients, resulting in hypertension and other renal problems. ${ }^{17}$ Six months after radiochemotherapy for gastric cancer, Jansen et al. observed a $20 \%$ decrease in the function of the left kidney. They believe that the renal impairment may increase over time. ${ }^{18}$

In 2001, at the Institute of Oncology Ljubljana, Slovenia, postoperative radiochemotherapy was established as a standard clinical practice in the treatment of patients who had undergone radical resection of non-metastatic gastric adenocarcinoma of stages Ib-IV. The short-term results of this treatment regimen have already been published. ${ }^{10}$ The aim of the present report is to present the long-term results of postoperative radiochemotherapy for gastric adenocarcinoma, including late treatment-related toxicity.

\section{Patients and methods}

Between 2001 and 2004, 123 patients (79 males, 44 females) with the mean age of 60 years locally and/or regionally. ${ }^{2-4} \mathrm{~A}$ number of studies have been conducted to improve the treatment outcome of these patients and, as a result, postoperative radiochemotherapy was established as a routine treatment in the USA as well as in other countries. ${ }^{6-17}$ The authors believe that the INT 0116 protocol is safe and acceptable for clinical use., , $^{3,8-11}$ (range: 31-76 years), were treated for gastric adenocarcinoma of TNM stages Ib-IV (nonmetastatic), with postoperative concomitant radiochemotherapy. One hundred and seven $(87 \%)$ patients had radical resection (R0) of the tumor, and in the remaining 16 (13\%) patients, non-radical (R1) resection was made. Distal subtotal, total, 
and multivisceral resection of the stomach was performed in $40.7 \%, 29.3 \%$, and $27.6 \%$ of patients, respectively, and three patients $(2.4 \%)$ had resection of the carcinoma on gastric stump. In $92(74.7 \%)$ patients, at least 15 lymph nodes were removed and histologically examined, in 27 (22\%) patients, less than 15 lymph nodes were examined, while for 4 (3.3\%) patients, no data on the lymph node status was available. Most frequently, the primary tumor originated in the antrum (38.2\%). Sixty-one percent of patients had advanced disease and $96.7 \%$ of patients had $\mathrm{N}+$ disease (Table 1).

After surgery, all patients with the disease of pathological stage Ib or more were presented to a multidisciplinary advisory team, consisting of a surgeon, radiation oncologist and medical oncologist, in order to assess the prospects of eventual adjuvant treatment. Eligibility of patients for adjuvant therapy was assessed with respect to the blood test results and performance status ( $\geq 2$ according to the World Health Organisation [WHO]). More extensive radiologic investigations already performed before surgery to rule out metastatic disease, were repeated only in the patients with clinically suspected progression of the disease.

During the therapy, the patients were clinically examined and referred for hematological and biochemical blood testing once a week. The therapy-related local and systemic toxicity was assessed according to the National Cancer Institute Common Toxicity Criteria (NCI-CTC) version 2.0. ${ }^{19}$ WHO scale was used to determine the performance status of patients and their body weight was measured on weekly basis.

Adjuvant treatment was initiated six weeks after surgery. It consisted of concomitantly applied chemotherapy and radiotherapy. In the chemotherapy part of the protocol, five cycles of 5 -FU $\left(425 \mathrm{mg} / \mathrm{m}^{2}\right)$ and LV (20 mg/m²) administered as five-day intravenous infusions were planned. The treatment cycle was repeated every 28 days. During radiotherapy, the intensity of chemotherapy was decreased. In the second and third cycle, only 4 and 3 applications of the drugs were administered, respectively. After the completed radiotherapy, the patients received two more five-day chemotherapy cycles.

Irradiation was applied during the second and third cycle of chemotherapy. The patients were irradiated on linear accelerator with 5-15 MV photon beams for 5 days per week, at a daily dose of $1.8 \mathrm{~Gy}$. The irradiation field involved the primary tumor site and regional lymph node areas with a safety margin of $1.5-2 \mathrm{~cm}$. Two opposite (AP-PA) beams were applied. Total irradiation dose was $45 \mathrm{~Gy}$ and total irradiation time 5 weeks.

After the completed treatment, followup examinations were performed every three months in the first two years and then at six month intervals to the end of five years. The patients were than referred back to their general practitioner's care and attended follow-up examinations only once per year. The follow-up examination consisted of clinical examination, complete blood count, liver and renal functional tests and measurements of CEA and Ca 19-9. Ultrasound (US) or computed tomography (CT) of the abdomen were performed every six months, chest radiography or CT once a year and endoscopic examination of the upper gastrointestinal tract as clinically indicated. During the follow-up period, any suspected disease relapse or recurrence was confirmed by biopsy.

\section{Statistics}

Statistical analysis was performed using personal computer and software statistical package SPSS, version 13 (SPSS Inc., USA). 
Table 2. Toxicity of adjuvant radiochemotherapy

\begin{tabular}{lllllll}
\hline \multirow{2}{*}{ Toxicity } & \multicolumn{7}{l}{ NCI grade (\%) } & & \\
\cline { 2 - 7 } & $\mathbf{0}$ & $\mathbf{1}$ & $\mathbf{2}$ & $\mathbf{3}$ & $\mathbf{4}$ & Total \\
\hline Stomatitis & 48 & 10.6 & 15.4 & 26 & 0 & 100 \\
Radiodermatitis & 95.2 & 1.6 & 1.6 & 1.6 & 0 & 100 \\
Diarrhoea & 79.7 & 5.7 & 5.7 & 8.9 & 0 & 100 \\
Dysphagia & 44.7 & 12.1 & 21.2 & 22 & 0 & 100 \\
Nausea, vomiting & 56.9 & 11.4 & 13 & 18.7 & 0 & 100 \\
Infection & 50.4 & 18.7 & 18.7 & 12.2 & 0 & 100 \\
Leukocyte count & 30.1 & 29.3 & 30.1 & 9.7 & 0.8 & 100 \\
Haemoglobin level & 19.5 & 70.7 & 9.8 & 0 & 0 & 100 \\
Platelet count & 92.7 & 7.3 & 0 & 0 & 0 & 100 \\
\hline
\end{tabular}

(median 17.6 weeks), whereas the duration of the radiotherapy part of the protocol ranged from 3.3-18 weeks (median 5.3 weeks). In regard to the intensity of radiotherapy and chemotherapy doses, $82 \%$ of patients completed the treatment according to the protocol. There was no treatment-related death. The frequency and severity of early toxic ef-

The main endpoints were as follows: locoregional control (LRC, the event was local and/or regional recurrence); diseasefree survival (DFS, the event was local, regional or systemic recurrence); the disease-specific survival (DSS, the event was death due to gastric adenocarcinoma), and overall survival (OS, the event was death from any cause).

The survival of patients was computed from the date of surgery to the $1^{\text {st }}$ of May, 2009 (close out date). Survival probability was calculated using Kaplan-Meier estimate $^{20}$, and log rank test ${ }^{21}$ was used to evaluate the differences between individual groups of patients. Independent prognostic values of variables that appeared statistically significant on univariate analysis were tested by multivariate Cox regression analysis model..$^{22}$ Two-sided tests were used and the differences at $p<0.05$ were considered as statistically significant.

\section{Results}

\section{Treatment outcome}

All 123 patients were evaluable for analysis. Postoperative chemotherapy started 3.6-11.9 weeks after surgery (median 5.9 weeks). Total postoperative treatment time ranged from 4.9- 32.6 weeks fects of radiochemotherapy are shown in Table 2. In regard to the late side effects, only renal functional impairment (the rise of creatinine level of grade 1 , but without hypertension or other renal impairment) was observed in 5 patients $(4.1 \%)$. The observed median time interval of creatinine level elevation was 10.5 months (range: 8.9-13.4 months).

On the close-out date, median followup time of all treated patients was 51.8 months (range: 5.3-96.4 months), with the median follow-up time of 64.5 months (range: 51.7-96.4 months) for 56 survivors. In $56(45.5 \%)$ patients, alive at the time of analysis, 53 had no signs of disease. Of $67(54.5 \%)$ dead patients, 60 died of gastric carcinoma, 4 of other causes (stroke, lung cancer, cancer of the caecum, myocardial infarction; $1 / 4$ patients with simultaneous locoregional recurrence), and in 3 patients, the cause of death could not be determined.

After adjuvant radiochemotherapy, disease re-appeared in $62(50.4 \%)$ patients. Local and/or regional recurrence developed in $7(5.7 \%)$ patients in the median time of 18 months (range: 9.7-56.3 months) after surgery. Locoregional failure and systemic dissemination were diagnosed in $11(8.9 \%)$ patients in the median time of 11 months (range: 6.2-25.7 months), and 


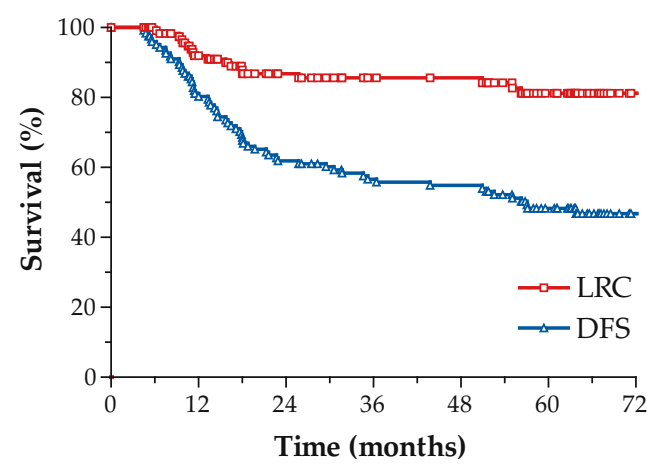

Figure 1. Locoregional control (LRC) and disease-free survival (DFS).

systemic metastases alone in 44 (35.8\%) patients in the median time of 16 months (range: 4.5-63.8 months). The 5-year LRC, DFS, DSS and OS were $81 \%, 48.3 \%, 50.4 \%$, and $48.4 \%$, respectively (Figures 1,2 ).

\section{Prognostic factors}

On univariate analysis of survival, locally advanced disease (pT3-4, pN3, overall TNM stage IV) was predictive for worse LRC and survival compared to early disease stages. In addition, poor outcome of patients was associated also with higher degree of stomach involvement with cancer (whole stomach vs. involvement of individual stomach areas), tumor location in the stomach (cardia vs. other subsites), Borrmann type 4, growth type according to Lauren (diffuse type vs. others), with the presence of vascular and perineurial invasion and intensity of chemotherapy ( $<5$ cycles vs. 5 cycles of chemotherapy) (in all instances $\mathrm{p}<0.05)$.

The multivariate analysis showed that the tumor involvement of cardia and low intensity of chemotherapy were independent adverse prognostic factors for DSS and OS. More advanced pT-stage and tumors with diffuse growth type according to Lauren were identified as negative independent prognostic factor for OS. They

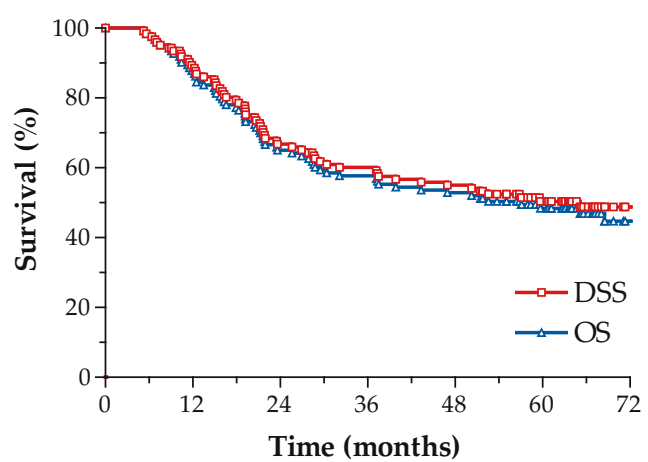

Figure 2. Disease-specific survival (DSS) and overall survival (OS).

were also on the threshold of statistical significance for DSS (Table 3).

\section{Discussion}

Short-term results of pooperative treatment with radiochemotherapy for gastric cancer in our population of patients have already been published. ${ }^{10}$ As some other authors, we may also conclude that the combined radiotherapy and chemotherapy with 5-FU is feasible, with acceptable toxicity, and seems to have a potential to improve treatment outcome compared to surgery as the sole mode of treatment for this poor prognosis group of patients. ${ }^{5-15}$ After a longer follow-up, excellent LRC and acceptable DFS, DSS and OS were confirmed, which concurs with the results from other studies. 5,6,8,9,11,13,23

In the present analysis, the patients with more advanced tumors, cardia involvement, perineurial and vascular invasion had poorer survival. With the prolongation of follow-up time, the diffuse growth type according to Lauren, Borrmann type 4 and low number of chemotherapy cycles, were also recognized as negative prognostic factors for disease outcome. All these factors are considered to be well established negative prognosticators in clinics for patients 
Table 3. Multivariate analysis of survival

\begin{tabular}{|c|c|c|c|c|c|}
\hline \multirow[t]{2}{*}{ Prognostic factors } & \multirow[t]{2}{*}{$\mathbf{n}$} & $\begin{array}{l}\text { Locoregional } \\
\text { control }\end{array}$ & $\begin{array}{l}\text { Disease } \\
\text { free } \\
\text { survival }\end{array}$ & $\begin{array}{l}\text { Disease } \\
\text { specific } \\
\text { survival }\end{array}$ & $\begin{array}{l}\text { Overall } \\
\text { survival }\end{array}$ \\
\hline & & $\mathbf{p}$ & $\mathrm{p}$ & $\mathrm{p}$ & $\mathrm{p}$ \\
\hline \multicolumn{6}{|l|}{ pT- stage } \\
\hline $\mathrm{pT} 1+2$ & 48 & & & 0.06 & 0.04 \\
\hline $\mathrm{pT} \mathrm{3+4}$ & 75 & & & & \\
\hline \multicolumn{6}{|l|}{$\mathrm{pN}$ - stage } \\
\hline $\mathrm{pN} 0+1+2$ & 98 & & & & \\
\hline $\mathrm{pN} 3$ & 25 & & & & \\
\hline \multicolumn{6}{|l|}{ Overall stage } \\
\hline Stage Ib -III & 93 & & & & \\
\hline Stage IV & 30 & & & & \\
\hline \multicolumn{6}{|l|}{$\begin{array}{l}\text { Stomach } \\
\text { involvement }\end{array}$} \\
\hline Whole stomach & 7 & & & & \\
\hline Individual areas & 116 & & & & \\
\hline \multicolumn{6}{|l|}{ Primary tumour site } \\
\hline Cardia & 16 & & & 0.01 & 0.01 \\
\hline Other sites & 107 & & & & \\
\hline
\end{tabular}

\begin{tabular}{ll}
\hline Perineurial invasion & \\
Yes & 45 \\
No & 59 \\
\hline
\end{tabular}

\begin{tabular}{ll}
\hline Angioinvasion & \\
Yes & 45 \\
No & 23 \\
\hline
\end{tabular}

\begin{tabular}{ll}
\hline Borrman type & \\
Type $1-3$ & 61 \\
Type 4 & 24 \\
\hline
\end{tabular}

\begin{tabular}{llll}
\hline $\begin{array}{l}\text { Growth type } \\
\text { according to Lauren }\end{array}$ & & & \\
Diffuse & 61 & 0.09 & 0.05 \\
Intestinal & 56 & & \\
\hline No of ChT cycles & & 0.03 & 0.04 \\
Less than 5 cycles & 24 & & \\
Five cycles & 99 & & \\
\hline pT - pathological T-stage; pN - pathological N-stage; ChT - chemotherapy.
\end{tabular}

with gastric cancer or with another malignancies and are also usually mentioned as such in pertinent literature. ${ }^{4,24-27}$ The subgroup of patients with early, distal tumors in whom distal subtotal resection was per- formed had no better outcome compared to those who had more advanced disease and, consequently, underwent more extensive surgery. This observation pointed out the potential of adjuvant therapy to neutralize otherwise well established prognostic power of higher tumor stage and tumor's localization. Contrary to our previous analysis $^{10}$, the dose of 5 -FU per cycle and pretreatment $\mathrm{Hb}$ concentration $\leq 110 \mathrm{~g} / 1$ lost their influence on survival. The reason for this finding could be longer follow up and consecutively more adequate statistical analysis.

The results of both multivariate analyses (i.e. the one from the past and the present one) exposed tumors located in the cardia as negative and independent prognosticators, which was pointed out also in other similar studies. ${ }^{26,27}$

In the present analysis, like at the most of analysis of other malignancies ${ }^{25}$, to no surprise, a more advanced pT-stage was established as negative independent prognostic factor for OS, while for DSS it was on the threshold of statistical significance. The patients who 
received less than five cycles of chemotherapy had worse DSS and OS. It is well known that the intensity of chemotherapy can have an influence on treatment outcome neoplasma. ${ }^{24,28}$ In our series of patients, we didn't notice other serious late toxicities than the rise of creatinine blood level of grade 1 . Jansen et al. observed a progressive decrease in the function of the left kidney which was $11 \%$ and $52 \%$ after 6 and 18 months, respectively. ${ }^{18}$ Due to the more accurate conformal radiotherapy planning, we hope there will be less late renal toxicity $^{29,30}$, however, to approve this, longer follow-up of our patients is needed.

Because of high incidence of unresectable disease and distant metastases, this issue is to be addressed in future prospective studies exploring new systemic drugs and regimens. We should pay greater attention to preoperative radiochemotherapy, which could be the best treatment approach also in this type of malignancy, as it is in rectal, esophageal and breast cancer ${ }^{31}$, although more randomized trials are needed to evaluate the survival benefits of this approach.

\section{Conclusions}

From the analysis of the treatment results of a group of 123 patients with operable gastric carcinoma and median follow up time of almost 5 years (more than 5 years in survivors), we may conclude that postoperative adjuvant radiochemotherapy with 5-FU and LV can efficiently improve the treatment outcome, particularly in regard to LRC, with acceptable early and late toxicity. Because of high incidence of distant metastases, this issue has to be addressed in well designed future prospective studies exploring new systemic drugs and regimens.

\section{References}

1. Gunderson LL, Burch PA, Donohue JH. The Role of Irradiation as a Component of Combined Modality Treatment for Gastric Carcinoma. J Infus Chemother 1995; 5: 117-24.

2. Gunderson LL, Sosin H. Adenocarcinoma of the stomach: Areas of failure in a reoperation series (second or symptomatic look) clinicopathological correlation and implications for adjuvant therapy. Int J Radiat Oncol Biol Phys 1982; 8: 1-11.

3. Smalley SR, Gunderson L, Tepper J, Martenson JA Jr, Minsky B, Willett C, et al. Gastric surgical adjuvant radiotherapy consensus report: rationale and treatment implementation. Int J Radiat Oncol Biol Phys 2002; 52: 283-93.

4. Willett CG, Gunderson LL. Stomach. In: Perez CA, Brady LW, editors. Principles and practice of radiation oncology, 5th edition. Philadelphia: Lippincott-Raven Publishers; 2008. p. 1318-35.

5. Macdonald JS, Smalley SR, Benedetti J, Hundahl SA, Estes NC, Stemmermann GN, et al. Chemoradiotherapy after surgery compared with surgery alone for adenocarcinoma of the stomach or gastroesophageal junction. N Engl J Med 2001; 345: 725-30.

6. Bora H, Unsal D, Akmansu M. Results of chemoirradiation after curative resection of locally advanced gastric cancer. Int J Clin Pract 2004; 58: 451-6.

7. Moertel CG, Childs DS, O'Fallon JR, Holbrook MA, Schutt AJ, Reitemeier RJ. Combined 5-fluorouracil and radiation therapy as a surgical adjuvant for poor prognosis gastric carcinoma. J Clin Oncol 1984; 2: 1249-54.

8. Hughes BG, Yip D, Chao M, Gibbs P, Carroll S, Goldstein D, et al. Audit of postoperative chemoradiotherapy as adjuvant therapy for resected gastroesophageal adenocarcinoma: an Australian multicentre experience. ANZ J Surg 2004; 74: 9516.

9. Park SH, Kim DY, Heo JS, Lim DH, Park CK, Lee $\mathrm{KW}$, et al. Postoperative chemotherapy for gastric cancer. Ann Oncol 2003; 14: 1373-7.

10. Oblak I, Velenik V, Anderluh F, Strojan P. Results of adjuvant radiochemotherapy for gastric adenocarcinoma in Slovenia. Eur J Surg Oncol 2007; 33: 982-7. 
11. Surenkok S, Beyzadeoglu M, Oysul K, Ozyigit G, Ataergin S, Arpaci F, et al. The management of gastric adenocarcinoma with postoperative chemoirradiation. A non-randomized comparison of oral UFT and 5-FU. Tumori 2008; 94: 70-4.

12. Oechsle K, Bokemeyer C, Hartmann JT, Budach W, Trarbach T, Stahl M, et al. Four consecutive multicenter phase II trials of adjuvant chemoradiation in patients with completely resected high-risk gastric cancer: the experience of the German AIO/ $\mathrm{ARO} / \mathrm{CAO}$ group. J Cancer Res Clin Oncol 2009; 135: 163-72.

13. Baeza MR, Giannini TO, Rivera SR, Gonzalez P, Gonzalez J, Vergara E, et al. Adjuvant radiochemotherapy in the treatment of completely resected, locally advanced gastric cancer. Int J Radiat Oncol Biol Phys 2001; 50: 645-50.

14. Beşe NS, Büyükünal E, Özgüro lu M, Demir G, Yildirim A, Mandel NM, et al. Toxicity and survival results of a phase II study investigating the role of postoperative chemoradioimmunotherapy for gastric adenocarcinoma. Strahlenther Onkol 2005; 181: 652-9.

15. Bleiberg H, Goffin JC, Dalesio O, Buyse M, Pector JC, Gignoux M, et al. Adjuvant radiotherapy and chemotherapy in resectable gastric cancer: A randomized trial of the gastro-intestinal tract cancer cooperative group of the EORTC. Eur J Surg Oncol 1989; 15: 535-43.

16. Lim DH, Kim DY, Kang MK, Kim YI, Kang WK, Park CK, et al. Patterns of failure in gastric carcinoma after D2 gastrectomy and chemoradiotherapy: a radiation oncology's view. $\mathrm{Br}$ J Cancer 2004; 91: 11-7.

17. Earle CC, Maroun J, Zuraw L; Cancer Care Ontario Practice Giudelines Initiative Gastrointestinal Cancer Disease Site Group. Neoadjuvant or adjuvant therapy for resectable gastric cancer? A practice guideline. Can J Surg 2002; 45: 438-46.

18. Jansen EP, Saunders MP, Boot H, Oppedijk V, Dubbelman R, Porritt B, et al. Prospective study on late renal toxicity following postoperative chemoradiotherapy in gastric cancer. Int $J$ Radiat Oncol Biol Phys 2007; 67: 781-5.

19. Ajani JA, Welch SR, Raber MN, Fields WS, Krakoff IH. Comprehensive criteria for assessing therapyinduced toxicity. Cancer Invest 1990; 8: 147-5920. Kaplan EL, Meier P. Nonparametric estimation from incomplete observations. J Am Stat Assoc 1958; 53: 457-81.
21. Peto R, Pike MC, Armitage P, Breslow NE, Cox DR, Howard SV, et al. Design and analysis of randomized clinical trials requiring prolonged observation of each patient. II. Analysis and examples. $\mathrm{Br}$ J Cancer 1977; 35: 1-39.

22. Cox DR. Regression models and life-tables. J R Stat Soc Bull 1972; 34: 187-220.

23. MacDonald JS. Role of post-operative chemoradiation in resected gastric cancer. J Surg Oncol 2005; 90: 166-70.

24. Kovač V, Smrdel U. Meta-analyses of clinical trials in patients with non-small cell lung cancer. Neoplasma 2004; 51: 334-40.

25. Debevec L, Jerič T, Kovač V, Bitenc M, Sok M. Is there any progress in routine management of lung cancer patients? A comparative analysis of an institution in 1996 and 2006. Radiol Oncol 2009; 43: 47-53.

26. Yao JC, Mansfield PF, Pisters PW, Feig BW, Janjan NA, Crane C, et al. Combined-modality therapy for gastric cancer. Semin Surg Oncol 2003; 21: 223-7.

27. Kim JP, Lee JH, Kim SJ, Yu HJ, Yang HK. Clinicopathologic characteristics and prognostic factors in 10783 patients with gastric cancer. Gastric Cancer 1998; 1: 125-33.

28. DeVita VT Jr, Chu E. Principles of Medical Oncology. In: DeVita VT Jr, Lawrence TS, Rosenberg SA, editors. Cancer: principle and practice of oncology, 8th edition. Philadelphia: Lippincott Williams and Wilkins; 2008. p. 337-50.

29. Kragelj B. Increased late urinary toxicity with whole pelvic radiotherapy after prostatectomy. Radiol Oncol 2009; 43: 88-96.

30. Stavrev P, Schinkel C, Stavreva N, Fallone BG. How well are clinical gross tumor volume DVHs approximated by an analytical function? Radiol Oncol 2009; 43: 132-5.

31. Velenik V. Locally recurrent rectal cancer: treatment options. Radiol Oncol 2009; 43: 144-51. 